\title{
FACTORES ASOCIADOS CON EL INICIO TEMPRANO DE LA ACTIVIDAD SEXUAL POR GÉNERO, EN LOS ESTUDIANTES DE LA I. E. P. MARISCAL CASTILLA - HUANCAYO
}

\section{FACTORS ASSOCIATED WITH EARLY ONSET OF SEXUAL ACTIVITY GENDER IN STUDENTS OF THE IEP MARISCAL CASTILLA - HUANCAYO}

\author{
Paredes Caballero, Magda', Adler Julio Soto Rojas. ${ }^{2}$, Perales Rojas Yessenia
}

\section{RESUMEN}

Se ha realizado la investigación en la I.E.P. Mariscal Castilla (Colegio mixto) de la Provincia de Huancayo, distrito de El Tambo, que puede ser considerada muestra representativa de la población adolescente, la adolescencia cobra gran significación por los múltiples y complejos cambios físicos, cognitivos y psicosociales que ocurren en esta etapa, como consecuencia, la sexualidad influye significativamente en el modo de vida de los adolescentes y repercute en la problemática de salud que puede aparecer en ese momento 0 en las sucesivas etapas del ciclo vital. Resulta impostergable, por lo tanto, considerar la sexualidad en el contexto de la salud integral de los adolescentes, por la repercusión que tiene en el estilo de vida, e incorporar como estrategia de intervención la educación de la sexualidad tempranamente y en el marco de la promoción y prevención continua de salud a través del ciclo vital. Las variables estudiadas fueron sexo, con quién viven, edad de inicio de las relaciones sexuales, con quién ha debutado sexualmente, medios de la adquisición de la información de métodos anticonceptivos conocidos, acerca de sexualidad. Los resultados obtenidos mostraron una disminución de la edad de inicio de las relaciones sexuales; la edad preponderante es sobre los 15 años de edad, pero se observa en porcentajes menores que ya existe iniciación de un acto sexual con otra persona, ya sea hombre o mujer, indiferentemente en menores de 13 a 14 años con un 30\% de los encuestados. En cuanto a los métodos anticonceptivos conocidos el que ocupa el mayor porcentaje es el condón y la abstinencia sexual, Los estudiantes. Una de las conclusiones a la que se llegó es que la principal vía de obtener los conocimientos acerca de sexualidad es mediante el colegio, familiares, amigos, personal de salud e internet.

Palabras clave: Adolescente, sexualidad, género

\section{ABSTRACT}

The investigationhas been carried out in the IEP Mariscal Castilla (mixed School) of Huancayo Province, district of El Tambo which can be considered as a representative sample of adolescent population. Adolescence gets great meaning by the multiple and complex cognitive, physical and psicosociales changes that occur in this phase, as a consequence, sexuality significantly influences the way of life of adolescents and results in the problems of health, that can appear just at that stage or in the successive phases of their life cycle. Therefore it turns out to be imposible passing over to consider sexuality in the context of integral health of adolescents, because of the repercussion it has in their way of life, and to incorporate it as an intervention strategy in the education of sexuality early and in the framework of the promotion and continuous prevention of health through the life cycle. The variables studied were sex, who do they live with, age of starting sexual relations, who has debuted sexually with, media of acquisition of information on known contraceptive methods. The results obtained showed a decrease of the age of startting sexual relations; the predominant age is 15 years, but it is observed in smaller percentages that initiation 1 paredesmagda@yahoo.com

2 maypaca@hotmail.com 
of a sexual act with another person happens in less than 13 to 14 years, whether man or woman. As for known contraceptive methods, the one that occupies the major percentage is condom and sexual abstinence. One of the arrived conclusions is that the main way to obtain the know how about sexuality is by means of school, family, friends, personnel of health and internet.

Keywords: Adolescent, sexuality, kin

\section{INTRODUCCIÓN}

La adolescencia es un período de transición entre la niñez y la edad adulta, comprendida entre los 10 y 19 años en el cual enfrentan múltiples y complejos cambios físicos, cognitivos y psicosociales que ocurren en esta etapa, los que determinan significados y formas de expresión diferentes de la sexualidad. Como consecuencia, la sexualidad influye significativamente en el modo de vida de los adolescentes y repercute en la problemática de salud que puede aparecer en ese momento 0 en las sucesivas etapas del ciclo vital.

La dinámica sexual en el curso de estos últimos 20 años, tanto en países desarrollados como en los subdesarrollados, ha cambiado considerablemente. La vida sexual de los jóvenes empieza cada vez más temprano, esto lleva consigo innumerables problemas, sobre todo relacionados a la salud reproductiva

En el Perú, como en muchos países, los y las adolescentes inician actividad sexual a edades más tempranas que en generaciones anteriores. El inicio sexual temprano está asociado también a un mayor número de parejas sexuales a lo largo de la vida. Estas tendencias tienen una influencia enorme en la ocurrencia de comportamientos sexuales de riesgo y en la epidemiología del embarazo adolescente, como también en las infecciones de transmisión sexual y VIH-SIDA.

Reducir el embarazo adolescente y las infecciones de transmisión sexual, incluida el SIDA, son políticas de salud pública en nuestro país, pero hasta ahora las estrategias han sido insuficientes. Hay estudios que muestran el inicio temprano de las relaciones románticas, el uso temprano de alcohol y otras drogas, son factores determinantes del inicio de la actividad sexual temprana
Entre los retos que deben enfrentar los adolescentes están los relacionados con la práctica de su sexualidad y el riesgo que esto conlleva para su salud reproductiva, por el posible advenimiento de embarazos no deseados, sus consecuencias, así como el riesgo de adquirir una Infección de Transmisión Sexual (ITS). El problema del inicio temprano de las relaciones sexuales en los adolescentes sin el debido conocimiento acerca del tema, se ha generalizado en el mundo de hoy y en nuestro país con el sistema de salud del MINSA, no se prioriza. Para algunos prestigiosos autores el papel de los medios masivos de difusión es trascendental en la incitación al inicio sexual precoz de los adolescentes.

El objetivo de este estudio es conocer cuáles son los factores sociales asociados con el inicio temprano de la actividad sexual por género.

\section{MATERIAL Y MÉTODOS}

El área de estudio comprende a los estudiantes de secundaria de la Institución Educativa Pública Mariscal Castilla del distrito de El Tambo Huancayo. La investigación es de tipo Correlacional simple, ya que permiten relacionar 0 asociar variables, es un diseño no experimental transversal, descriptivo simple analítico, cuantitativo. y estadístico tiene como objetivo Determinar los factores asociados con el inicio temprano de la actividad sexual por género, en los y las estudiantes del $4^{\circ}$ y $5^{\circ}$ grado de secundaria la I. E. P. Mariscal Castilla - Huancayo.

Población y muestra: El universo de la población estudiantil del $4^{\circ}$ y $5^{\circ}$ grado de secundaria es de: 1,360 alumnos. Muestra. El valor de $n=176.56=177$ 
Es decir la muestra estadística es de 177 alumnos, que han sido determinados al azar, en aulas completas. (40 estudiantes).

El instrumento que se utilizó fue la encuesta orientada a conocer el inicio temprano de la actividad sexual según género y sus factores asociados a ello.

La recolección de datos fue tomada de aula en aula, previa coordinación con el Profesor Coordinador de OTOE y los profesores tutores. Esta técnica se utilizó para relacionar las variables dependientes e independientes vinculado a los factores que determinan el inicio temprano de la actividad sexual según género (hombres y mujeres), del $4^{\circ}$ y $5^{\circ}$ grado de secundaria de la I.E.P. Mariscal Castilla.

\section{RESULTADOS}
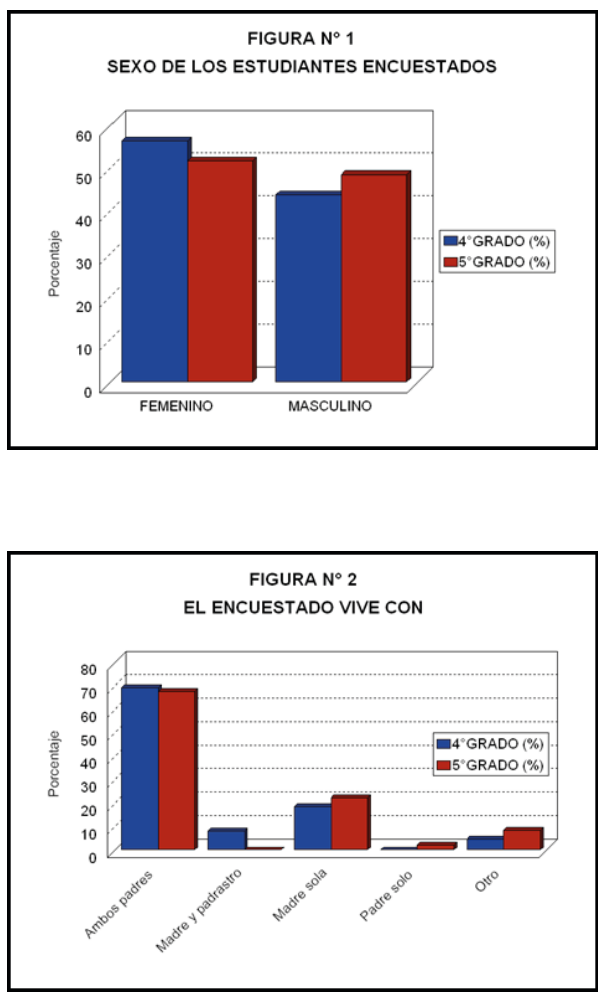
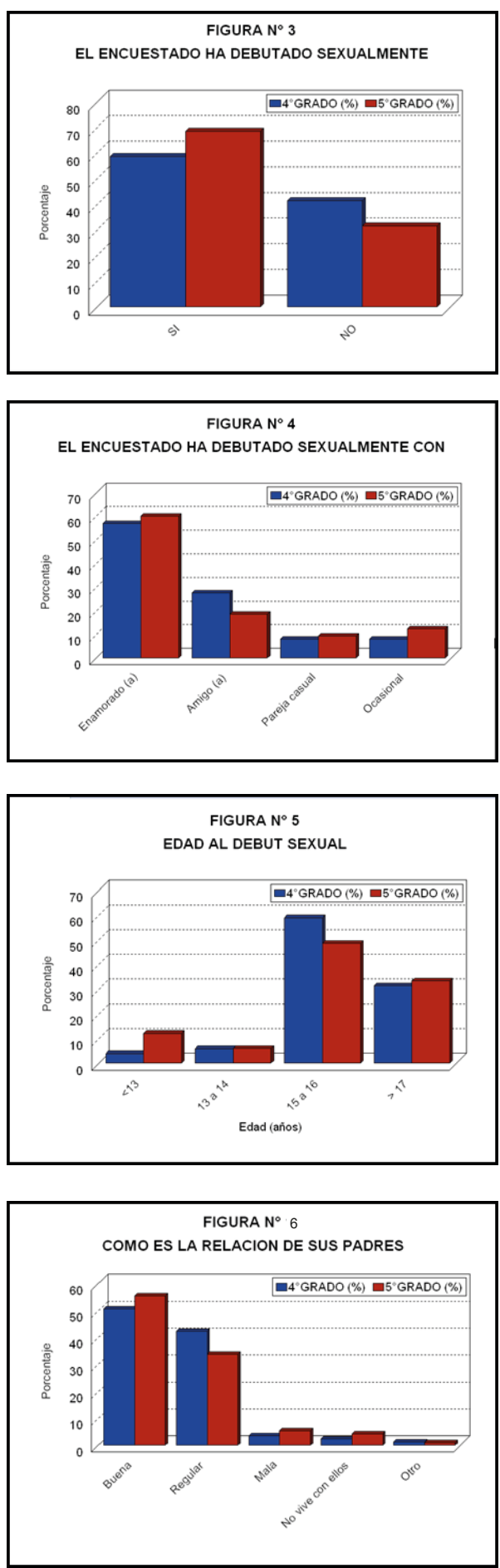

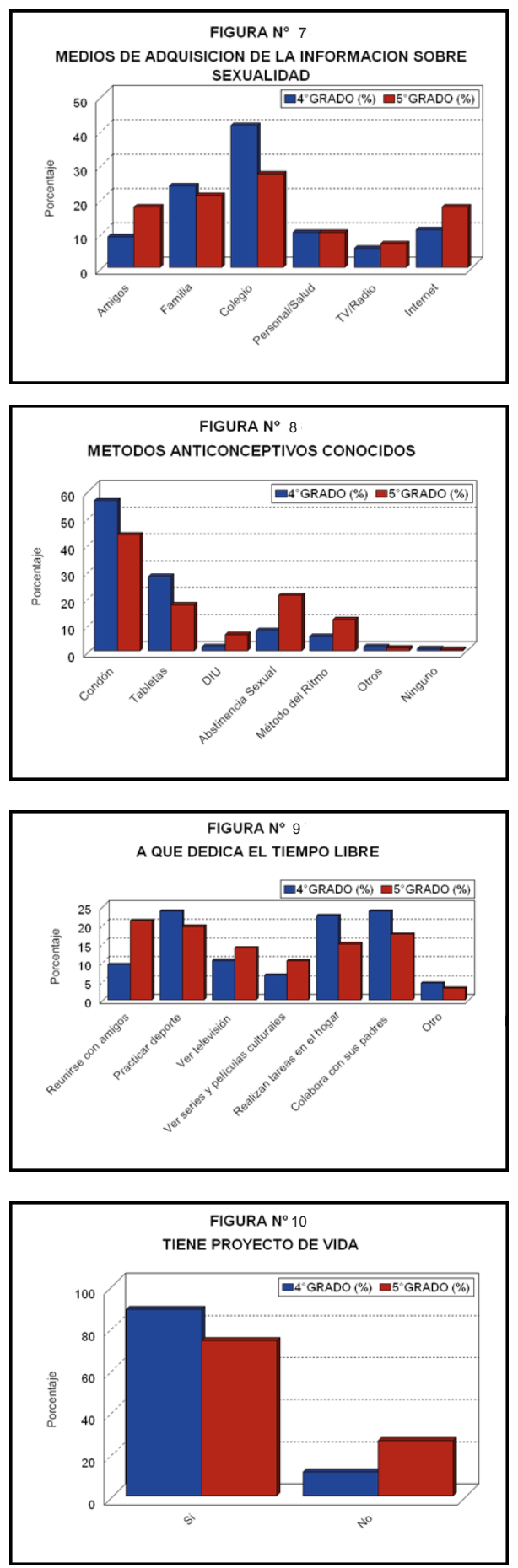

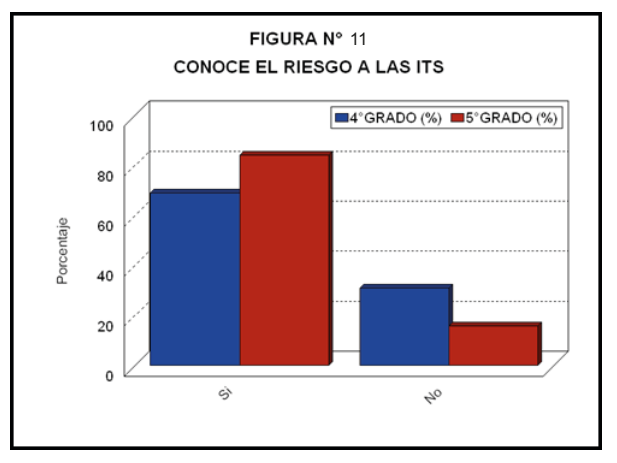

\section{DISCUSION}

Contrastando los resultados obtenidos en las encuestas a los estudiantes del $4^{\circ}$ y $5^{\circ}$ Grado de Secundaria de la I. E. P. Mariscal Castilla - Huancayo para determinar los factores asociados con el inicio temprano de la actividad sexual por género, el adolescente es el individuo que acaba de terminar la niñez y que transcurre desde la pubertad hasta el completo desarrollo del organismo, es un período de múltiples cambios. En ésta investigación se observó que el porcentaje de encuestados correspondió al grupo etario entre 15 y 19 años de edad, el sexo predominante en $4^{\circ}$ Grado y $5^{\circ}$ Grado fue el femenino, alrededor del $70 \%$ de los encuestados viven con ambos padres, debemos de indicar que más de la mitad el $60 \%$ de los mismos han debutado sexualmente, determinándose que el sexo no es factor determinante ya que el porcentaje es similar para ambos sexos, la edad preponderante es sobre los 15 años de edad, pero se observa en porcentajes menores que ya existe iniciación de un acto sexual con otra persona, ya sea hombre 0 mujer, indiferentemente en menores de 13 a 14 años con un $30 \%$ de los encuestados.

Como confirma González LI, Miyar PE. Infertilidad y sexualidad dice que estudios realizados en diferentes grupos de adolescentes muestran que la edad de las primeras relaciones sexuales ha disminuido notablemente y que cerca del $60 \%$ ha tenido como mínimo una relación sexual a los 13 años, han debutado sexualmente un $60 \%$ en ambos Grados con el enamorado (a) podemos observar que no solamente existe empatías instintivas sino que éste inicio sexual está relacionado a afectos y sentimientos de adolescentes, es preocupante el alto porcentaje $16 \%$ del $4^{\circ}$ Grado ha debutado sexualmente 
con pareja casual/ocasional y el $31 \%$ del $5^{\circ}$ Grado. Sobre antecedentes de la madre el 52\% si tuvo hijo antes de los 20 años. Al referirse a los medios de adquisición de información sobre sexualidad en $4^{\circ}$ Grado y $5^{\circ}$ Grado alrededor del $40 \%$ lo adquirió en el colegio, seguido por amigos y personal de salud e internet.

En cuanto a los métodos anticonceptivos conocidos el que ocupa el mayor porcentaje es el condón y la abstinencia sexual, Los estudiantes del $4^{\circ}$ Grado encuestados el $89 \%$ tiene un Proyecto de vida y del $5^{\circ}$ Grado el 74\%. El mayor porcentaje de ambos Grados conoce el riesgo de la ITS, Por último el $4^{\circ}$ y $5^{\circ}$ Grado considera tener una autoestima alta y media.

Maddaleno, M. y E. N. Suárez Ojeda:"situación social de los adolescentes y jóvenes en América Latin ", en La salud del adolescente y el joven OPS №.552 dice " Entre los retos que deben enfrentar los adolescentes están los relacionados con la práctica de su sexualidad y el riesgo que esto conlleva para su salud reproductiva, por el posible advenimiento de embarazos no deseados, sus consecuencias, así como el riesgo de adquirir una Infección de Transmisión Sexual (ITS). Los adolescentes y adultos jóvenes constituyen hoy día alrededor del 30\% de la población mundial. Esto concuerda con el resultado demostrado en la fig, 19 se observa que más del 70 \% conoce el riesgo de las Infecciones de transmisión sexual ITS.

\section{CONCLUSION}

1. El grupo etario que predominó entre los encuestados fue el de 15 a 19 años y el sexo fue el femenino.

2. Los resultados obtenidos mostraron una disminución de la edad de inicio de las relaciones sexuales; la edad preponderante es sobre los 15 años de edad,

3. Es significativo la mayor cantidad de adolescentes que comenzaron sus relaciones sexuales antes de los 12 años en el grupo etario entre 12 y 14 años, determinándose que el sexo no es factor determinante para inicio sexual, ya que el porcentaje es similar para ambos sexos,

4. Es preocupante el alto porcentaje $16 \%$ del $4^{\circ}$ Grado ha debutado sexualmente con pareja casual/ ocasional y el $31 \%$ del $5^{\circ}$ Grado

5. Los métodos anticonceptivos más conocidos fundamentalmente es el condón, abstinencia sexual y el uso de tabletas.
6. La vía fundamental de adquirir conocimientos los adolescentes acerca de sexualidad es en el colegio, a través de sus familiares, amigos, internet quedando el personal de salud en el último lugar.

\section{REFERENCIAS BIBLIOGRÁFICAS}

1. Campo-A. Adalberto, Silva D. José .(2004) Factores asociados con el inicio temprano de relaciones sexuales en estudiantes adolescentes de un colegio de Bucaramanga, Colombia-Vol. 33

2. GüeL Pedro, Goldstein Eduardo (2000); Orientaciones normativas y conversaciones sobre intimidad, actividad sexual. En: Estudio Nacional de Comportamiento Sexual Primeros Análisis: Chile Ministerio de Salud. Santiago. Chile.2003

3. Henriques-M. María Elena y Yunes Joao. (1993). Adolescencia: Equivocaciones y Esperanzas. En: Género Mujer y Salud en las Américas. Organización Panamericana de la Salud, Washington DC, Estados Unidos.

4. Molina S. Marta, Ferrada N, Cristina; Perez V. Ruth ET AL. (2004). Embarazo en la adolescencia y su relación con la deserción escolar. Revista Médica de Chile. vol.132.

5. Padilla M. (2001) Salud sexual de los y las adolescentes en El Salvador. REVSOGIA

6. Palma Irma. (2001). Salud y derechos sexuales y reproductivos de adolescentes y jóvenes en el contexto de la reforma de salud en Chile. 11 de Agosto 2013 www.paho.org/English/HDP/HDW/adolescents

7. Quintana S. Alicia y Vásquez D. Ernesto. (2003). Introducción, construcción de la identidad de género y abuso sexual. En: Construcción social de la sexualidad adolescente. Género y salud sexual. Tercera Edición. Instituto de Educación y Salud. Lima. Perú.

8. Santana P. Felipe. (2006) Asociación entre algunos factores psicosociales y el inicio de las relaciones sexuales en adolescentes escolares. Revista Cubana Med Gen Integr.

9. Tarazona C. David. (2005). Estado del arte sobre comportamiento sexual adolescente. En: Revista Electrónica del Instituto Psicología y Desarrollo 2006.

10. Zeidenstein Sondra y Moore Kirsten. (1999). Introducción. En: Aprendiendo sobre sexualidad. Una manera de comenzar. Santiago Chile. Traducción realizada por el Instituto Chileno de Medicina Reproductiva. 